\title{
Multimodal vibration damping using a simplified current blocking shunt circuit
}

\author{
Ghislain Raze ${ }^{1}$, Ahmad Paknejad ${ }^{2}$, Guoying Zhao ${ }^{2}$, Christophe Collette ${ }^{1,2}$ and Gaetan \\ Kerschen ${ }^{1}$
}

\begin{abstract}
The tuning of a simplified current blocking shunt circuit able to mitigate the vibration amplitude of multiple structural resonances is addressed in this paper. The proposed strategy exploits the two-port network formalism in combination with physically-motivated approximations to tune sequentially the electrical elements of the different branches of the shunt circuit. The resulting tuning method does not resort to optimization algorithms and requires only the knowledge of quantities that are easy to measure experimentally. It is demonstrated both numerically and experimentally using a piezoelectric beam.
\end{abstract}

\section{Keywords}

Piezoelectric absorber, Multimodal damping, Current blocking shunt circuit, Two-port network, Piezoelectric beam

\section{Introduction}

Piezoelectric shunt damping is a popular technique for passive vibration control. A piezoelectric transducer bonded to a structure transforms though the piezoelectric effect part of the mechanical energy into electrical energy. The electrical energy can then be dissipated using resistive elements shunting the transducer. Inductive elements can also be used to create an electrical resonance with the inherent piezoelectric capacitance, thereby enhancing energy dissipation. Hagood and von Flotow (1991) laid down the theoretical foundations of resistive and series RL shunt circuits. As an alternative, $\mathrm{Wu}(1996)$ proposed to connect a parallel RL shunt circuit to the transducer. Caruso (2001) demonstrated that series and parallel RL shunt circuits are nearly equivalent in terms of vibration attenuation. Thomas et al. (2012) provided alternative tuning rules and assessed vibration mitigation performance thanks to the effective electromechanical coupling coefficient. The exact $H_{\infty}$-optimal solution corresponding to the minimization of the maximum amplitude of the frequency response function of a single-degree-of-freedom (SDOF) oscillator was found by Soltani et al. (2014) for the series RL shunt circuit. It was derived for parallel RL shunt circuits by Ikegame et al. (2019), by noting the analogy of this problem with series RC shunts for electromagnetic transducers (Tang et al. 2016).

In practice, engineering structures possess multiple vibration modes, which renders the tuning procedure more complicated than when considering the single mode assumption. Berardengo et al. (2016) showed that the frequencydependent character of the piezoelectric capacitance due to the electromechanical interaction (see, e.g., de Marneffe (2007)) is to be taken into account for improved accuracy. The significant impact on performance of non-resonant modes was illustrated by Høgsberg and Krenk (2017), and an explicit correction for background flexibility and inertia was proposed. Gardonio and Casagrande (2017) showed that simple lumped-parameter models yield inaccurate tuning of the shunt parameters for a simply-supported plate. The near-equivalence of using the effective electromechanical coupling coefficient and a residual-mode corrected electromechanical coupling coefficient when tuning shunt circuit parameters was demonstrated by Toftekær et al. (2018).

An advantage of piezoelectric absorbers is their conceptually simple extension to the control of multiple resonances. A first approach is to use as many shunted transducers as resonances to be controlled. Examples where this approach was considered include beams (Viana and Steffen, Jr 2006) and truss-cored sandwich panels (Guo and Jiang 2014). The recent work of Toftekær and Høgsberg (2020) proposed explicit corrections to account for non-resonant modes and cross-interaction between the different shunt circuits.

A second approach for multimodal piezoelectric shunt damping is based on multiple patches interconnected through an electrical network. The network is designed to be the electrical analog of the mechanical structure to which it is coupled (Alessandroni et al. 2002; Giorgio et al. 2015). This tuning strategy was validated experimentally, e.g., on plate structures (Lossouarn et al. 2016).

A third approach uses a single transducer, as multiple transducers cannot always be accommodated for practical reasons (Moheimani and Fleming 2006). Since the classical $\mathrm{RL}$ shunt resonates with the transducer at a specific

\footnotetext{
${ }^{1}$ University of Liège, Liège, Belgium

${ }^{2}$ Université Libre de Bruxelles, Brussels, Belgium

\section{Corresponding author:}

Ghislain Raze

Space Structures and Systems Laboratory (S3L), Department of Aerospace and Mechanical Engineering, University of Liège

Quartier Polytech 1 (B52/3)

Allée de la Découverte 9

Liège, B-4000, Belgium

Email: g.raze@uliege.be
} 
frequency, one can devise a more complex electrical circuit that resonates at multiple frequencies. Edberg et al. (1991) tested this concept experimentally for two modes; the circuit topology was later generalized by Hollkamp (1994) to control an arbitrary number of resonances. In view of the difficulty to tune the circuit, $\mathrm{Wu}$ (1998) introduced the so-called current blocking shunt circuit, which, however, requires a large number of electrical components. Behrens et al. (2003) proposed a current flowing shunt circuit and Fleming et al. (2003) developed a series-parallel impedance structure requiring a lower number of components. The downside of these latter topologies is that they provide a rather low vibration reduction on the controlled modes. The aforementioned shunt circuits are discussed in more details in Moheimani and Fleming (2006). More recently, ladder topologies were proposed by Agneni et al. (2006) and Goldstein (2011).

Closed-form solutions for multimodal vibration damping with a single transducer suffer from important approximations, yielding sub-optimal designs. This is why Fleming et al. (2002) resorted to gradient-based optimization to minimize the $\mathrm{H}_{2}$ norm of a transfer function in order to determine the resistances of multiple-branch shunt circuits. Jeon (2009) used particle swarm optimization to tune a seriesparallel impedance-type circuit whereas Cigada et al. (2012) also exploited gradient-based algorithms to tune a current flowing shunt circuit. More recently, Berardengo et al.(2017) obtained the optimal impedance through optimization based on linear matrix inequalities. An interesting feature of this work is that the circuit topology is not assumed beforehand, but it is rather an outcome of the optimization process. Despite its versatility, numerical optimization suffers from important drawbacks. First, it requires an accurate model of the structure to be controlled, which is not often available in practice. Second, there is no guarantee that the objective function is convex, and the global minimum can be difficult to reach.

In this context, the present paper introduces a noniterative, model-less strategy for tuning the electrical parameters of the simplified current blocking shunt circuit in (Agneni et al.2006). This circuit, represented in Figure 1, is made of cascade connections of different stages comprising each a shunt branch $Z_{i}$ and a $L C$ filter that provides some degree of independence between the stages. Nonetheless, owing to their electrical interconnections, the different shunt branches of the circuit interact with each other. To account for this interaction during the tuning process, the circuit preceding a specific shunt branch is abstracted out as a two-port network (Alexander and Sadiku 2000). With this formalism, we propose to identify an equivalent electromechanical structure such that a so-called fictitious shunt branch can be tuned using the formulas available in the literature. The parameters of the physical shunt branch are then deduced from those of the fictitious shunt branch and of the two-port network. Once the considered shunt branch has been tuned, the cascade structure is exploited for tuning the next branch of the current blocking shunt circuit. The process is repeated until the last shunt branch $Z_{N}$ has been tuned. The tuning procedure proposed in this work improves upon the ones proposed previously in the literature $(\mathrm{Wu} 1998$; Agneni et al. 2006) because it accounts automatically for the influence of non-resonant modes and assesses correctly the electromechanical coupling between the shunt branches and the structure.

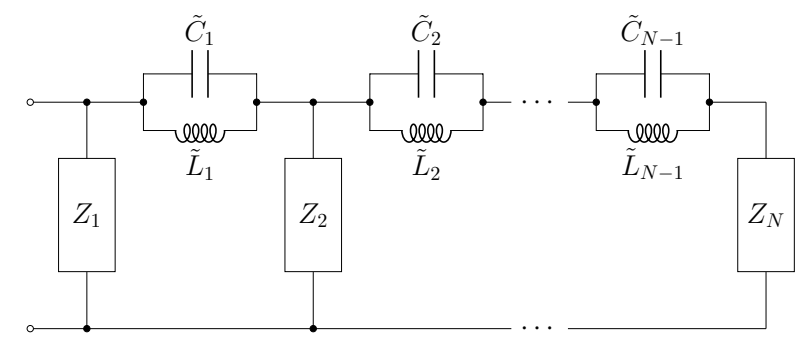

Figure 1. Simplified current blocking shunt circuit Agneni et al. 2006).

The paper is organized as follows. The basics of Resonant piezoelectric shunt damping are first briefly reviewed and are used to tune a Shunt branch connected to an electrical network. To create a shunt circuit that works at multiple frequencies, Multimodal shunt damping with a single transducer is discussed. The proposed procedure for tuning a Simplified current blocking shunt circuit is then introduced. The approach is demonstrated both numerically and experimentally with two Applications Conclusion on this work is finally drawn.

\section{Resonant piezoelectric shunt damping}

The equations governing the behavior of a SDOF springmass system coupled to a piezoelectric stack are given by (Hagood and von Flotow 1991)

$$
\left\{\begin{array}{rl}
\left(m s^{2}+k_{o c}\right) x-\theta q_{p} & =f \\
\frac{1}{C_{p}^{\varepsilon}} q_{p}-\theta x & =-V_{p}
\end{array},\right.
$$

where $m$ is the mass, $k_{o c}$ is the structural stiffness when the piezoelectric transducer is open-circuited, $\theta$ is a coupling coefficient, and $C_{p}^{\varepsilon}$ is the piezoelectric capacitance at constant strain. $x$ represents the displacement of the mechanical system, $f$ is an external force, $q_{p}$ is the charge flowing through the piezoelectric transducer, $V_{p}$ is the voltage across its electrodes, and $s$ is the Laplace variable. The coupling present between the mechanical and electrical variables allows to design a shunt circuit connected to the electrodes of the transducer in order to modify the dynamics of the structure. Mathematically, this imposes a relation between the variables $V_{p}$ and $q_{p}$. Typically, series RL and parallel RL circuits are used to create an electrical resonance that interacts with the mechanical resonance if the circuit is properly tuned.

The open-circuit $\left(q_{p}=0\right)$ and short-circuit $\left(V_{p}=0\right)$ structural resonance frequencies are given by

$$
\omega_{o c}^{2}=\frac{k_{o c}}{m}, \quad \omega_{s c}^{2}=\frac{k_{s c}}{m}=\frac{k_{o c}-\theta^{2} C_{p}^{\varepsilon}}{m},
$$

respectively. Assuming an unforced system $(f=0)$ and condensing the first line of Equation (1) into the second one, the inverse of the dynamic capacitance (de Marneffe 2007), i.e., the dynamic elastance, is obtained as

$$
\frac{V_{p}}{q_{p}}=-\frac{1}{C_{p}^{\varepsilon}} \frac{s^{2}+\omega_{s c}^{2}}{s^{2}+\omega_{o c}^{2}} .
$$


The knowledge of $C_{p}^{\varepsilon}, \omega_{s c}$ and $\omega_{o c}$ is thus sufficient to tune the parameters of a shunt circuit. In the literature, the shunt parameters are often expressed as a function of the effective electromechanical coupling factor (EEMCF) whose square is given by (Hagood and von Flotow 1991)

$$
K_{c}^{2}=\frac{\omega_{o c}^{2}-\omega_{s c}^{2}}{\omega_{s c}^{2}}
$$

The EEMCF can also be used as an indirect measure of the expected attenuation performance (de Marneffe 2007, Thomas et al. 2012, Soltani et al. 2014).

For the minimization of the maximum vibration amplification of the host structure, i.e., the minimization of the $H_{\infty}$ norm of the frequency response function (FRF), the optimal parameters for series and parallel RL shunts are given in the Appendix The resulting FRF exhibits two peaks of equal amplitude, comparatively low with respect to the uncontrolled structure (when the transducer is either shortor open-circuited), as illustrated in Figure 2 for a series RL shunt circuit (Soltani et al. 2014).

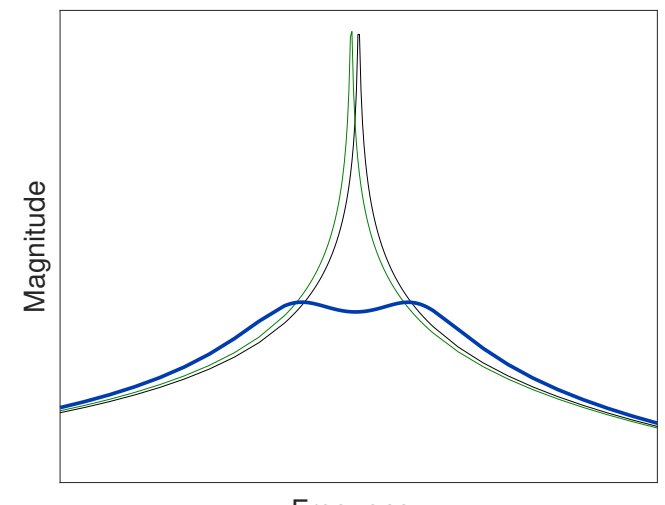

Frequency

Figure 2. FRF a SDOF spring-mass system with a short-circuited transducer (-), an open-circuited transducer $(-)$ and a series $R L$ piezoelectric shunt circuit $(-)$.

\section{Shunt branch connected to an electrical network}

In the previous section, the shunt branch was directly connected to the piezoelectric transducer. This section considers the case where an additional electrical network $\mathbf{G}$ is present between the shunt and the transducer, as in Figure 3. The electrical network is modeled using the twoport network theory (Alexander and Sadiku 2000).

\section{Dynamic elastance and capacitance seen from the shunt branch}

Assuming that the two-port network is composed of linear elements, the electrical quantities at the piezoelectric transducer and at the shunt branch can be related through a transfer matrix $\mathbf{G}$ :

$\left[\begin{array}{c}V_{s} \\ s q_{s}\end{array}\right]=\mathbf{G}(s)\left[\begin{array}{c}V_{p} \\ s q_{p}\end{array}\right]=\left[\begin{array}{ll}g_{11}(s) & g_{12}(s) \\ g_{21}(s) & g_{22}(s)\end{array}\right]\left[\begin{array}{c}V_{p} \\ s q_{p}\end{array}\right]$.

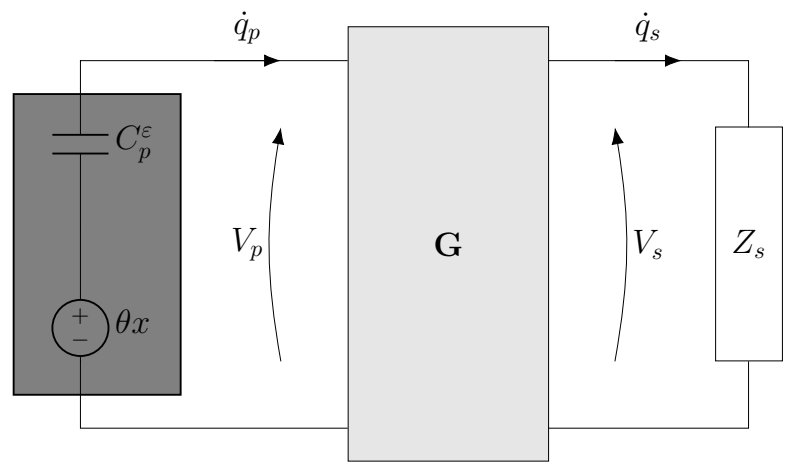

Figure 3. Shunt branch $Z_{s}$ connected to a piezoelectric transducer through an electrical network $\mathbf{G}$.

If the network has no dependent sources, it is reciprocal, and its transfer matrix has a unit determinant (Alexander and Sadiku 2000). Forming the ratio of the two lines of Equation (5), one gets

$$
\frac{V_{s}}{q_{s}}=s \frac{g_{12}(s)+\frac{V_{p}}{s q_{p}} g_{11}(s)}{g_{22}(s)+\frac{V_{p}}{s q_{p}} g_{21}(s)}
$$

Depending on the considered transfer matrix, the dynamic elastance (6) could in principle represent any transfer function, but the simplicity of this expression illustrates one of the driving reasons for the use of the two-port network formalism in this work. It is assumed in this study that this elastance is similar to that in Equation (3) so that a piezoelectric shunt branch can be used. In other words, around the frequency of the mode to be damped, Equation (6) is approximated as

$$
\frac{V_{s}}{q_{s}} \approx-\frac{1}{\widehat{C}_{p}^{\varepsilon}} \frac{s^{2}+\widehat{\omega}_{s c}^{2}}{s^{2}+\widehat{\omega}_{o c}^{2}}
$$

to identify the characteristics $\widehat{C}_{p}^{\varepsilon}, \widehat{\omega}_{s c}$ and $\widehat{\omega}_{o c}$ of an equivalent dynamic elastance seen from the shunt branch. Similarly to the EEMCF defined in Equation (4), from $\widehat{\omega}_{s c}$ and $\widehat{\omega}_{o c}$, it is possible to form a modified EEMCF $\widehat{K}_{c}$, termed branch EEMCF (BEEMCF)

$$
\widehat{K}_{c}^{2}=\frac{\widehat{\omega}_{o c}^{2}-\widehat{\omega}_{s c}^{2}}{\widehat{\omega}_{s c}^{2}} .
$$

This BEEMCF is related to the considered shunt branch and assesses the electromechanical coupling existing between this branch and the structure. It may differ from the EEMCF because of the presence of a network. As shown in the examples, the BEEMCF has the same predictive capability on performance as the EEMCF in the nominal case; it is therefore a useful quantity to monitor.

As exemplified in Figure 1, the network can be composed of capacitive, resistive and inductive elements. Equation (7) is generally not able to model this accurately. A broader class of impedances can be represented if one augments the model given in Equation (7), by taking into account an inductance $\widehat{L}$ and a resistance $R$, as

$$
\frac{V_{s}}{q_{s}} \approx-\widehat{L} s^{2}-\widehat{R} s-\frac{1}{\widehat{C}_{p}^{\varepsilon}} \frac{s^{2}+\widehat{\omega}_{s c}^{2}}{s^{2}+\widehat{\omega}_{o c}^{2}} .
$$


$\widehat{L}$ and $\widehat{R}$ are lumped representations of the inductive and resistive elements of the network, respectively, enabling to approximate more accurately the dynamics of complex networks.

Alternatively to the dynamic elastance, one could form its inverse, the dynamic capacitance

$$
\frac{q_{s}}{V_{s}}=\frac{1}{s} \frac{g_{22}(s)+\frac{V_{p}}{s q_{p}} g_{21}(s)}{g_{12}(s)+\frac{V_{p}}{s q_{p}} g_{11}(s)}
$$

Considering the lumped reluctance $\widehat{B}$ and conductance $\widehat{G}$ of the network, the dynamic capacitance is approximated as

$$
\frac{q_{s}}{V_{s}} \approx-\frac{\widehat{B}}{s^{2}}-\frac{\widehat{G}}{s}-\widehat{C}_{p}^{\varepsilon} \frac{s^{2}+\widehat{\omega}_{o c}^{2}}{s^{2}+\widehat{\omega}_{s c}^{2}} .
$$

As shown in Figure 4, we adopt the idea of an equivalent piezoelectric structure made of the cascade connection of the piezoelectric transducer and the capacitive part of the network G (Caruso 2001; de Marneffe 2007; Berardengo et al. 2017). This equivalent system is fully defined by the parameters $\widehat{C}_{p}^{\varepsilon}, \widehat{K}_{c}$ and $\widehat{\omega}_{o c}$. The so-called fictitious shunt circuit comprises the shunt branch $Z_{s}$ and the inductiveresistive part of the network $\mathbf{G}$, i.e., $\widehat{L}$ (or $\widehat{B}$ ) and $\widehat{R}$ (or $\widehat{G}$ ). Before using these parameters to tune the shunt branch, an identification procedure is needed which is illustrated in the next section.

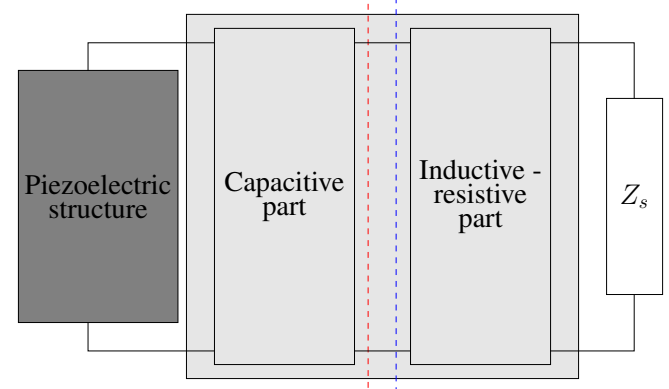

Equivalent piezoelectric structure Fictitious optimal shunt circuit

Figure 4. Schematic representation of the approach adopted in this work.

\section{Network identification}

The identification procedure starts with the determination of the best model for the network, i.e., either elastive or capacitive. In the former case, the parameters of Equation (9) are fitted to approximate the true elastance given in Equation (6). In the latter case, the parameters of Equation (11) are fitted to approximate the true capacitance given in Equation (10). Equation (9) results in elastances with four zeros and two poles, whereas Equation (11) results in elastances with four zeros (two of which are at $s=0$ ) and four poles. To discriminate between these two cases and select the best model, a criterion based on the distance from a frequency of interest $\omega_{0}$ (e.g., the resonance frequency with a short-circuited transducer $\omega_{s c}$ ) to the poles or zeros of the elastance is used. Let $d_{p, 1}$ and $d_{p, 2}$ be the distance from $j \omega_{0}$ to the closest poles and $d_{z, 1}$ and $d_{z, 2}$ be the distance from $j \omega_{0}$ to the closest zeros. Then,
1. If $d_{p, 1}+d_{p, 2}>d_{z, 1}+d_{z, 2}$, the network is assumed to be of elastive type

2. If $d_{p, 1}+d_{p, 2}<d_{z, 1}+d_{z, 2}$, the network is assumed to be of capacitive type

Dynamic elastance identification The method seeks an approximation

$$
\begin{aligned}
& \frac{V_{s}}{q_{s}} \approx-\widehat{L} s^{2}-\widehat{R} s-\frac{1}{\widehat{C}_{p}^{\varepsilon}} \frac{s^{2}+\widehat{\omega}_{s c}^{2}}{s^{2}+\widehat{\omega}_{o c}^{2}} \\
& \approx-\frac{\widehat{L} s^{4}+\widehat{R} s^{3}+\left(\widehat{L} \widehat{\omega}_{o c}^{2}+\frac{1}{\widehat{C}_{p}^{\varepsilon}}\right) s^{2}+\widehat{R} \widehat{\omega}_{o c}^{2} s+\frac{\widehat{\omega}_{s c}^{2}}{\widehat{C}_{p}^{\varepsilon}}}{s^{2}+\widehat{\omega}_{o c}^{2}}
\end{aligned}
$$

Inspired from the importance of poles and zeros in the feedback control theory, the approximation function

$$
\frac{V_{s}}{q_{s}} \approx \frac{\left(a s^{2}+b s+c\right)\left(s^{2}-2 z_{r} s+z^{2}\right)}{s^{2}-2 p_{r} s+p^{2}}
$$

keeps one pole $p$ and one zero $z$ of the actual $V_{s} / q_{s}$ (Equation (6)), the ones closest to the frequency of interest $\omega_{0}$. This sets two conditions on the parameters of the approximation function. The remaining conditions are merely fitting conditions.

The identification of the parameters goes as follows:

1. The closest pole (of magnitude $p$ and real part $p_{r}$ ) of $V_{s} / q_{s}$ to $j \omega_{s c}$ is selected. Then, $\widehat{\omega}_{o c}=p$.

2. The closest zero (of magnitude $z$ and real part $z_{r}$ ) of $V_{s} / q_{s}$ to $p$ is selected.

3. A function $f(s)=\left(s^{2}-2 p_{r} s+p^{2}\right) /\left(s^{2}-2 z_{r} s+\right.$ $\left.z^{2}\right) V_{s} / q_{s}$ is formed, and constants $a, b$ and $c$ are determined such that $\Im(f(s)), \Re(f(s))$ and $\Re(d f(s) / d s)$ are interpolated at $s=j \widehat{\omega}_{o c}$. For an experimentally-measured $V_{s} / q_{s}, a, b$ and $c$ are determined according to a least-squares error over a frequency interval containing $p$ and $z$.

4. The parameters $\widehat{L}, \widehat{R}, \widehat{\omega}_{s c}$ and $\widehat{C}_{p}^{\varepsilon}$ are identified by equating Equations 12 and 13 .

\section{Dynamic capacitance identification An approximation}

$$
\begin{aligned}
& \frac{q_{s}}{V_{s}} \approx-\frac{\widehat{B}}{s^{2}}-\frac{\widehat{G}}{s}-\widehat{C}_{p}^{\varepsilon} \frac{s^{2}+\widehat{\omega}_{o c}^{2}}{s^{2}+\widehat{\omega}_{s c}^{2}} \\
& \approx-\frac{\widehat{C}_{p}^{\varepsilon} s^{4}+\widehat{G} s^{3}+\left(\widehat{B}+\widehat{C}_{p}^{\varepsilon} \widehat{\omega}_{o c}^{2}\right) s^{2}+\widehat{G} \widehat{\omega}_{s c}^{2} s+\widehat{B} \widehat{\omega}_{s c}^{2}}{s^{2}\left(s^{2}+\widehat{\omega}_{s c}^{2}\right)}
\end{aligned}
$$

is sought under the form

$$
\frac{q_{s}}{V_{s}} \approx \frac{\left(a s^{2}+b s+c\right)\left(s^{2}-2 z_{r} s+z^{2}\right)}{s^{2}\left(s^{2}-2 p_{r} s+p^{2}\right)}
$$

to keep one pole $p$ and one zero $z$ of the actual $q_{s} / V_{s}$ (Equation (10). The identification method is summarized as follows:

1. The closest pole (of magnitude $p$ and real part $p_{r}$ ) of $q_{s} / V_{s}$ to $j \omega_{o c}$ is selected. Then, $\widehat{\omega}_{s c}=p$. 
2. The closest zero (of magnitude $z$ and real part $z_{r}$ ) of $q_{s} / V_{s}$ to $p$ is selected.

3. A function $f(s)=s^{2}\left(s^{2}-2 p_{r} s+p^{2}\right) /\left(s^{2}-2 z_{r} s+\right.$ $\left.z^{2}\right) q_{s} / V_{s}$ is formed, and constants $a, b$ and $c$ are determined such that $\Im(f(s)), \Re(f(s))$ and $\Re(d f(s) / d s)$ are interpolated at $s=j \widehat{\omega}_{s c}$. For an experimentally-measured $V_{s} / q_{s}, a, b$ and $c$ are determined according to a least-squares error over a frequency interval containing $p$ and $z$.

4. The parameters $\widehat{B}, \widehat{G}, \widehat{\omega}_{o c}$ and $\widehat{C}_{p}^{\varepsilon}$ are identified by equating Equations (14) and (15).

Example A host system governed by Equation (1) is considered, and its parameters are listed in Table 1] The network identification procedure is illustrated with the example of an LC circuit placed in parallel of the shunt branch, as depicted in Figure 5 , with $\tilde{L}=1.5 \mathrm{H}$ and $\tilde{C}=$ $1.5 \mathrm{~F}$.

\begin{aligned} Parameter & Value \\ \hline$m(\mathrm{~kg}) & 1 \\ k_{o c}(\mathrm{~N} / \mathrm{m}) & 1 \\ \theta(\mathrm{N} / \mathrm{C}) & 0.1 \\ C_{p}^{\varepsilon}(\mathrm{F}) & 1\end{aligned}$

Table 1. Parameters of the SDOF host system.

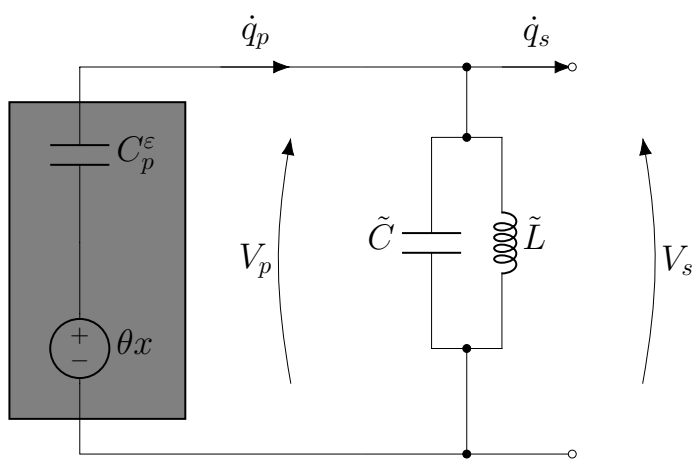

Figure 5. Parallel LC branch placed in parallel with the shunt branch.

The transfer function $V_{s} / q_{s}$, i.e., the true dynamic elastance, has two complex conjugate pairs of zeros and two complex conjugate pairs of poles. This transfer function as well as the frequencies of its poles and zeros are plotted in Figure 6. Since $d_{p, 1}+d_{p, 2}<d_{z, 1}+d_{z, 2}$, the best model is a capacitive-type network (in this case, this is an exact approximation), which is also confirmed by comparing the fitted dynamic elastances to the true ones.

\section{Shunt branch parameters}

Once the electrical network has been identified, the fictitious shunt circuit in Figure 4 is tuned such that it behaves as an optimal shunt branch from the equivalent piezoelectric structure's viewpoint. The corresponding shunt parameters $L^{\star}$ and $R^{\star}$ can thus be computed from the knowledge of $\widehat{C}_{p}^{\varepsilon}, \widehat{K}_{c}$ and $\widehat{\omega}_{o c}$ (or $\widehat{\omega}_{s c}$ ) using the formulas given in the Appendix

The physical shunt parameters $L$ and $R$ are then deduced from the knowledge of $L^{\star}, R^{\star}, \widehat{L}$ (or $\widehat{B}$ ) and $\widehat{R}$ (or $\widehat{G}$ ). The formulas in Table 2 depend on the type of identified

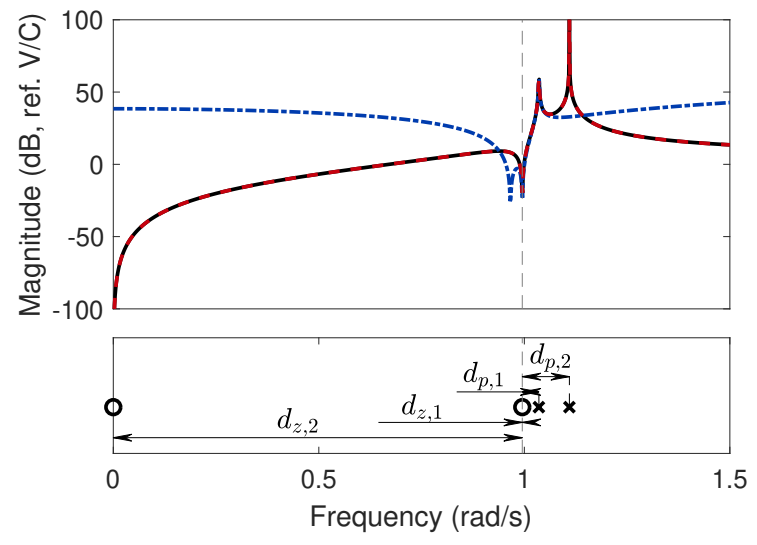

Figure 6. True dynamic elastance (-), fitted dynamic elastance assuming an elastive-type network (---) and fitted dynamic elastance assuming a capacitive-type network (- -). Poles $(\times)$ and zeros $(\circ)$ of the true dynamic elastance are indicated below the magnitude plot, and the frequency of interest $\omega_{0}=\omega_{s c}$ is given for reference (- -).

network and on the desired shunt branch (i.e., series or parallel RL). These formulas make the cascade connection of the inductive-resistive part of the network and the shunt branch have an impedance equal to the optimal one (at $\widehat{\omega}_{o c}$ for the series RL case and $\widehat{\omega}_{s c}$ for the parallel RL case).

\section{Shunt circuit impedance}

The shunt circuit impedance seen from the piezoelectric transducer can easily be obtained thanks to the two-port network formalism. Inverting Equation (5),

$$
\begin{aligned}
{\left[\begin{array}{c}
V_{p} \\
s q_{p}
\end{array}\right]=\mathbf{G}^{-1}(s)\left[\begin{array}{c}
V_{s} \\
s q_{s}
\end{array}\right] } & \\
= & {\left[\begin{array}{ll}
h_{11}(s) & h_{12}(s) \\
h_{21}(s) & h_{22}(s)
\end{array}\right]\left[\begin{array}{c}
V_{s} \\
s q_{s}
\end{array}\right] . }
\end{aligned}
$$

Since the voltage and charge of the shunt branch are related by

$$
\frac{V_{s}}{s q_{s}}=Z_{s}(s)
$$

the impedance $Z(s)$ of the shunt circuit is obtained by forming the ratio of the two lines of Equation (16) as

$$
Z(s)=\frac{V_{p}}{s q_{p}}=\frac{h_{11}(s) Z_{s}(s)+h_{12}(s)}{h_{21}(s) Z_{s}(s)+h_{22}(s)} .
$$

\section{Examples}

The host system governed by the parameters listed in Table 1 is considered once again. $R_{0}=0.123 \Omega$ and $L_{0}=1 \mathrm{H}$ are the resistance and inductance of the optimal shunt circuit when it is directly connected to the piezoelectric transducer. They are used to normalize the obtained resistance and inductance when presenting the results.

Two cases, namely a capacitor placed in parallel with the shunt branch and a capacitor placed in series with it, are studied. It is well known that such capacitive elements affect the electromechanical coupling (Caruso 2001; Fleming et al. 2003, de Marneffe 2007, Berardengo et al. 2018). 


\begin{tabular}{|c|c|c|}
\hline & Elastance & Capacitance \\
\hline & & $\widehat{\omega}_{o c}^{2} L^{\star}\left(1-L^{\star} \widehat{B}\right)-\left(R^{\star}\right)^{2} \widehat{B}$ \\
\hline Series shunt & $\begin{array}{l}L=L^{\star}-\widehat{L} \\
R=R^{\star}-\widehat{R}\end{array}$ & $\begin{array}{c}L=\frac{\overline{\widehat{\omega}_{o c}^{2}\left(1-R^{\star} \widehat{G}-L^{\star} \widehat{B}\right)^{2}+\left(\widehat{\omega}_{o c}^{2} L^{\star} \widehat{G}-R^{\star} \widehat{B}\right)^{2}}}{\widehat{\omega}_{o c}^{2} R^{\star}\left(1-R^{\star} \widehat{G}\right)-\widehat{\omega}_{o c}^{4}\left(L^{\star}\right)^{2} \widehat{G}}\end{array}$ \\
\hline Parallel shunt & $\begin{aligned} L & =\frac{\left(\widehat{R} R^{\star}-\widehat{\omega}_{s c}^{2} \widehat{L} L^{\star}\right)^{2}+\left(\widehat{L} R^{\star}+\widehat{R} L^{\star}-L^{\star} R^{\star}\right)^{2} \widehat{\omega}_{s c}^{2}}{\widehat{\omega}_{s c}^{2}\left(R^{\star}\right)^{2}\left(L^{\star}-\widehat{L}\right)-\widehat{\omega}_{s c}^{4}\left(L^{\star}\right)^{2} \widehat{L}} \\
R & =\frac{\left(\widehat{R} R^{\star}-\widehat{\omega}_{s c}^{2} \widehat{L} L^{\star}\right)^{2}+\left(\widehat{L} R^{\star}+\widehat{R} L^{\star}-L^{\star} R^{\star}\right)^{2} \widehat{\omega}_{s c}^{2}}{\widehat{\omega}_{s c}^{2}\left(L^{\star}\right)^{2}\left(R^{\star}-\widehat{R}\right)-\widehat{R}\left(R^{\star}\right)^{2}}\end{aligned}$ & $\begin{array}{c}R=\overline{\widehat{\omega}_{O C}^{2}\left(1-R^{\star} \widehat{G}-L^{\star} \widehat{B}\right)^{2}+\left(\widehat{\omega}_{O C}^{2} L^{\star} \widehat{G}-R^{\star} \widehat{B}\right)^{2}} \\
L=\frac{L^{\star}}{1-L^{\star} \widehat{B}} \\
R=\frac{R^{\star}}{1-R^{\star} \widehat{G}}\end{array}$ \\
\hline
\end{tabular}

Table 2. Tuning formulas: from optimal to physical shunt parameters.

Parallel capacitor A capacitor of capacitance $C_{p}$ is connected in parallel to the shunt branch. The transfer relation is given by

$$
\left[\begin{array}{c}
V_{s} \\
s q_{s}
\end{array}\right]=\left[\begin{array}{cc}
1 & 0 \\
-s C_{p} & 1
\end{array}\right]\left[\begin{array}{c}
V_{p} \\
s q_{p}
\end{array}\right] .
$$

Inserting this relation into Equation 10 and identifying the parameters in Equation (11) yield

$$
\begin{array}{rlrl}
\widehat{L}=0, & \widehat{R}=0, & \widehat{C}_{p}^{\varepsilon} & =C_{p}^{\varepsilon}+C_{p}, \\
\widehat{\omega}_{s c}=\omega_{s c}, & \widehat{\omega}_{o c}=\sqrt{\frac{C_{p} \omega_{s c}^{2}+C_{p}^{\varepsilon} \omega_{o c}^{2}}{C_{p}^{\varepsilon}+C_{p}}}
\end{array}
$$

i.e., a parallel capacitor affects the electromechanical coupling via a change in the open-circuit resonance frequency. After simple manipulations, the BEEMCF is found to be

$$
\widehat{K}_{c}=\frac{K_{c}}{\sqrt{1+\frac{C_{p}}{C_{p}^{\varepsilon}}}}
$$

which is in complete agreement with the enhanced modal electromechanical coupling factor defined in Berardengo et al. (2018).

For further validation, the obtained results are also compared to those of a brute-force numerical optimization of the $H_{\infty}$ norm of the compliance of a controlled SDOF system. A perfect agreement can be observed in Figure 7

Series capacitor A capacitor with capacitance $C_{s}$ is now connected in series with the shunt branch:

$$
\left[\begin{array}{c}
V_{s} \\
s q_{s}
\end{array}\right]=\left[\begin{array}{cc}
1 & -\frac{1}{s C_{s}} \\
0 & 1
\end{array}\right]\left[\begin{array}{c}
V_{p} \\
s q_{p}
\end{array}\right] .
$$

Inserting this relation into Equation (10) and identifying the parameters in Equation (11) yield

$$
\begin{aligned}
\widehat{L}=0, \quad \widehat{R}=0, \quad \widehat{C}_{p}^{\varepsilon}=\frac{C_{p}^{\varepsilon} C_{s}}{C_{p}^{\varepsilon}+C_{s}}, \\
\widehat{\omega}_{s c}=\sqrt{\frac{C_{s} \omega_{s c}^{2}+C_{p}^{\varepsilon} \omega_{o c}^{2}}{C_{p}^{\varepsilon}+C_{s}}}, \quad \widehat{\omega}_{o c}=\omega_{o c},
\end{aligned}
$$

i.e., a series capacitor affects the electromechanical coupling via a change in the short-circuit resonance frequency. The BEEMCF is

$$
\widehat{K}_{c}=\frac{K_{c}}{\sqrt{1+\left(1+K_{c}^{2}\right) \frac{C_{p}^{\varepsilon}}{C_{s}}}} .
$$

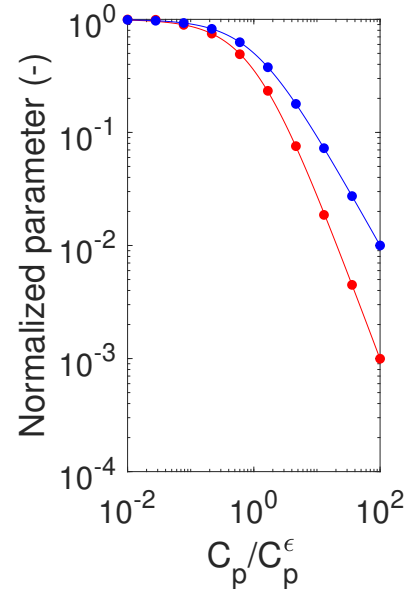

(a)

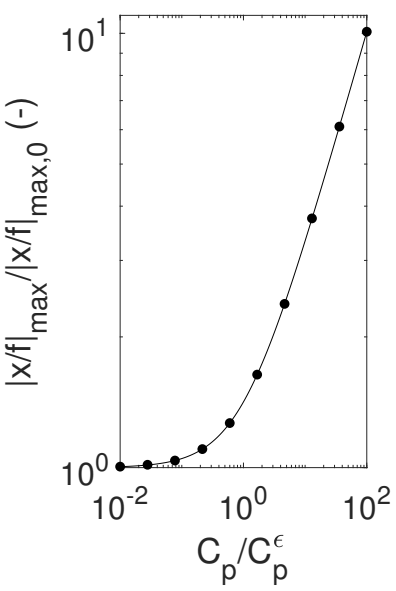

(b)
Figure 7. Evolution of the normalized shunt parameters (red: resistance, blue: inductance) (a) and of the maximum normalized amplitude (b) as functions of the normalized parallel capacitance. $\bullet$ : numerical optimization, —: Berardengo et al. 2018/proposed method.

The formula is not exactly the same as the enhanced modal electromechanical coupling factor given in Table 1 of Berardengo et al. (2018) because of the definition of the coupling factor, defined as $\sqrt{\left(\widehat{\omega}_{o c}^{2}-\widehat{\omega}_{s c}^{2}\right) / \omega_{s c}^{2}}$ therein. The slight difference is the term in $K_{c}^{2}$ which is important only for structures with high coupling coefficients.

Figure 8 evidences that the proposed method is in excellent agreement with numerical optimization and Berardengo et al. (2018). As shown in the inset of Figure 8|(b), our method is somewhat closer to the reference result given by numerical optimization.

\section{Multimodal shunt damping with a single transducer}

Shunt circuits targeting multimodal damping are discussed in the remainder of this paper. To create a shunt circuit that works at multiple frequencies, one could imagine a circuit with a parallel arrangement of shunt branches. However, to damp a mode at frequency $\omega$, the Appendix shows that the inductance and resistance scale as $\omega^{-2}$ and $\omega^{-1}$, respectively. Hence, the impedance of a shunt branch increases when the tuning frequency is decreased with the result that lower-frequency branches are by-passed and become ineffective.

In order to ensure that the current flows through the appropriate shunt branch at the appropriate frequency, 


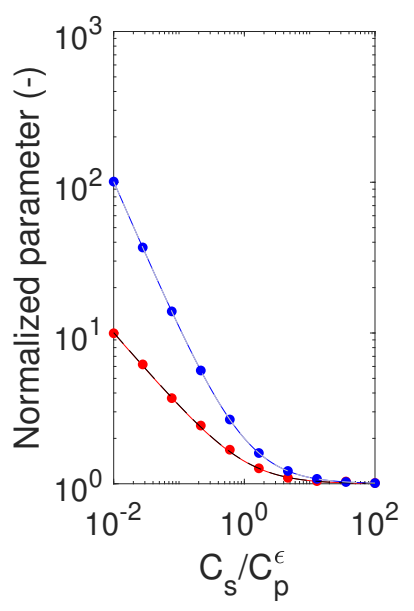

(a)

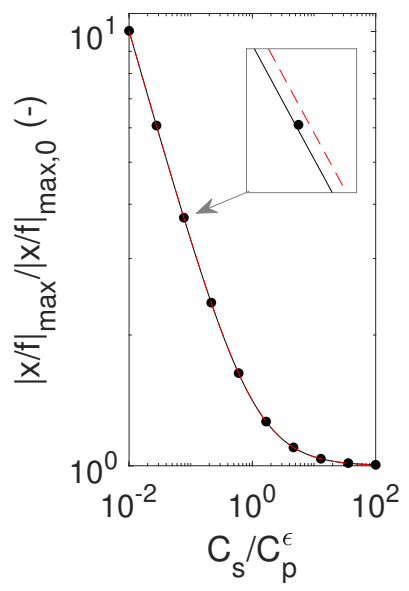

(b)
Figure 8. Evolution of the normalized shunt parameters (red/black: resistance, blue/gray: inductance) (a) and of the maximum normalized amplitude (b) as functions of the normalized series capacitance. - : numerical optimization, - -: formula from Berardengo et al. (2018), - : proposed method.

current bandpass or bandstop filters were introduced in multi-resonant shunt circuits. The current blocking shunt circuit originally proposed by $\mathrm{Wu}(1998)$ is shown in Figure 9. If $N$ resonances are targeted, each parallel branch contains $N-1$ current blocking (parallel LC) filters and one shunt branch (parallel RL). Considering a specific shunt branch targeting a particular structural frequency, the current blocking filters in the other parallel branches are such that they present an infinite impedance at this frequency, so that the current is forced to flow through this branch. A shortcoming of this architecture is that the required number of electrical components grows quadratically with the number of controlled resonances, which complicates the practical implementation (Moheimani and Fleming 2006).

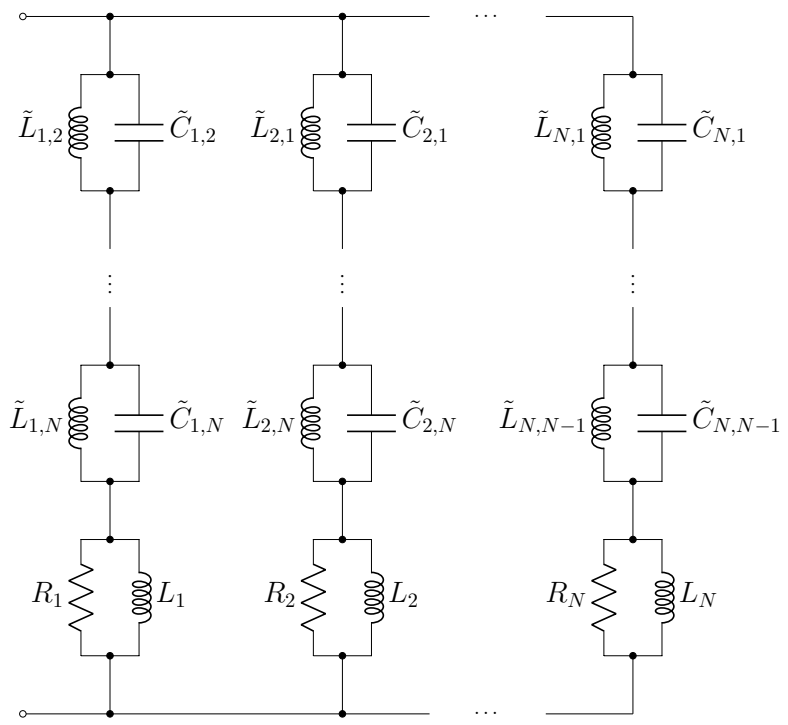

Figure 9. Current blocking shunt circuit $|\mathrm{Wu}| 1998)$.

Figure 1 depicts the layout of the simplified current blocking shunt circuit introduced in Agneni et al.(2006). The circuit is composed of ladder arrangement of repeated stages, each consisting of either a series or parallel RL circuit and a current blocking filter. The current blocking filter is tuned so as to force current to flow through the adequate shunt branch (and possibly the previous stages) and to prevent it from flowing through the next stages which present lower impedances.

As it will be shown in the Applications the existing tuning rules for these circuits (Wu 1998; Agneni et al. 2006) often yield sub-optimal parameters. In the next section, a new noniterative, model-less tuning strategy is developed.

\section{Simplified current blocking shunt circuit \\ Dynamic elastance seen from the piezoelectric transducer}

Multiple-degree-of-freedom piezoelectric structures can be modeled with the finite element method (Hagood et al. 1990; Thomas et al. 2009). For a structure with one piezoelectric transducer (or multiple transducers electrically connected so as to have one port), the electromechanical equations read

$$
\left\{\begin{array}{rl}
\mathbf{M} \ddot{\mathbf{x}}+\mathbf{C} \dot{\mathbf{x}}+\mathbf{K}_{o c} \mathbf{x}-\boldsymbol{\Theta} q_{p} & =\mathbf{f} \\
\frac{1}{C_{p}^{\varepsilon}} q_{p}-\boldsymbol{\Theta}^{T} \mathbf{x} & =-V_{p}
\end{array},\right.
$$

where $\mathbf{M}, \mathbf{C}$ and $\mathbf{K}_{o c}$ are structural mass, damping and (open-circuit) stiffness matrices, respectively, $\Theta$ is a piezoelectric coupling vector, $\mathbf{x}$ is the vector of generalized mechanical DOFs and $\mathbf{f}$ the conjugated generalized load vector.

Assuming an unforced structure $(\mathbf{f}=\mathbf{0})$, Laplacetransforming the first line of Equation (25) and condensing it into the second line, a relation between $V_{p}$ and $q_{p}$ is obtained

$$
\frac{V_{p}}{q_{p}}=-\frac{1}{C_{p}^{\varepsilon}}+\boldsymbol{\Theta}^{T}\left(\mathbf{M} s^{2}+\mathbf{C} s+\mathbf{K}_{o c}\right)^{-1} \boldsymbol{\Theta}
$$

Equation (26) is the dynamic elastance seen from the piezoelectric transducer. It can be obtained experimentally with an impedance analyzer or by measuring the capacitance at high frequency and identifying the open- and short-circuit resonance frequencies. If the latter approach is adopted, the dynamic elastance may conveniently be approximated by (de Marneffe 2007)

$$
\frac{V_{p}}{q_{p}} \approx-\frac{1}{C_{p}^{\varepsilon}}\left(1-\sum_{n=1}^{N} \frac{K_{c, i}^{2} \omega_{s c, i}^{2}}{s^{2}+2 \zeta_{i} \omega_{o c, i} s+\omega_{o c, i}^{2}}\right),
$$

where $\omega_{s c, i}$ and $\omega_{o c, i}$ are the short-circuit and open-circuit resonance frequencies of the $i^{\text {th }}$ mode, respectively, and $K_{c, i}$ is the EEMCF of that mode.

\section{Dynamic elastance seen from a specific shunt branch of the current blocking circuit}

We now show how to obtain the transfer matrix when twoport networks are connected in cascade, as in Figure 10

According to the conventions used in Figure 11|(a) the input and output quantities are related through the transfer matrix $\mathbf{G}_{s}$ as

$$
\left[\begin{array}{c}
V_{\text {out }} \\
s q_{\text {out }}
\end{array}\right]=\mathbf{G}_{s}(s)\left[\begin{array}{c}
V_{\text {in }} \\
s q_{\text {in }}
\end{array}\right]
$$




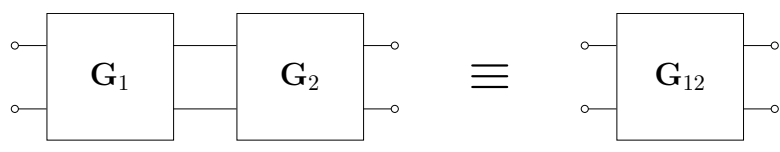

Figure 10. Cascade connection of two-port networks.

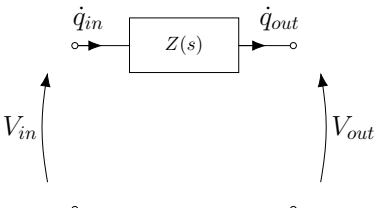

(a)

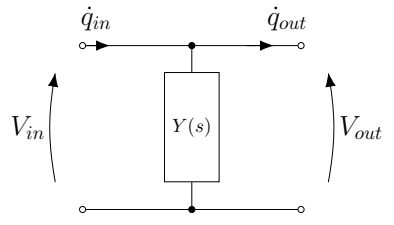

(b)
Figure 11. Two-port representation of series (a) and parallel (b) electrical elements.

The transfer matrix of a series element of impedance $Z(s)$ as in Figure 11|(a) is given by

$$
\mathbf{G}_{s}(s)=\left[\begin{array}{cc}
1 & -Z(s) \\
0 & 1
\end{array}\right] .
$$

Similarly, the transfer matrix of a parallel element of admittance $Y(s)$ as in Figure 11 (b) is

$$
\mathbf{G}_{p}(s)=\left[\begin{array}{cc}
1 & 0 \\
-Y(s) & 1
\end{array}\right] .
$$

For the simplified current blocking shunt circuit, the impedance of the $n^{\text {th }}$ current blocking filter is

$$
Z_{f, n}(s)=\frac{\tilde{L}_{n} s}{\tilde{L}_{n} \tilde{C}_{n} s^{2}+1} .
$$

The admittance of a series RL shunt branch is given by

$$
Y_{s, n}(s)=\frac{1}{L_{n} s+R_{n}},
$$

while that of a parallel RL shunt branch is

$$
Y_{p, n}(s)=\frac{1}{L_{n} s}+\frac{1}{R_{n}} .
$$

The transfer matrix of a cascade of $n$ two-port networks can be computed as the (ordered) matrix product of the transfer matrices of the connected networks (Alexander and Sadiku 2000)

$$
\mathbf{G}_{1 n}=\mathbf{G}_{n} \ldots \mathbf{G}_{2} \mathbf{G}_{1} \text {. }
$$

Eventually, Formulas (29), (30) and (34) can be used with either (26) or (27) to compute the dynamic elastance (6) and the dynamic capacitance (10) seen from a specific shunt branch of the simplified current blocking shunt circuit. This computation is readily implementable with a computer, which is another incentive to use the two-port network approach.

\section{Sequential tuning of the shunt circuit}

The tuning procedure is schematized in Figure 12 with its flowchart presented in Figure 13 At stage $n \neq N$, four parameters are to be determined, namely the filter, $\tilde{L}_{n}$ and $\tilde{C}_{n}$, and shunt, $L_{n}$ and $R_{n}$, parameters.
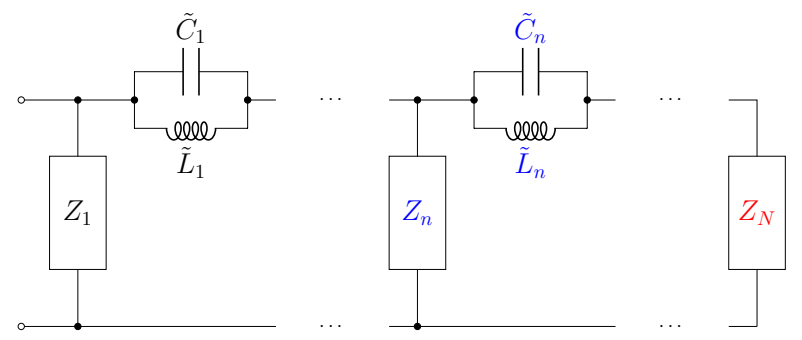

Figure 12. Tuning procedure for stage $n$ : quantities in black are known, quantities in blue are to be tuned and quantities in red are unknown.

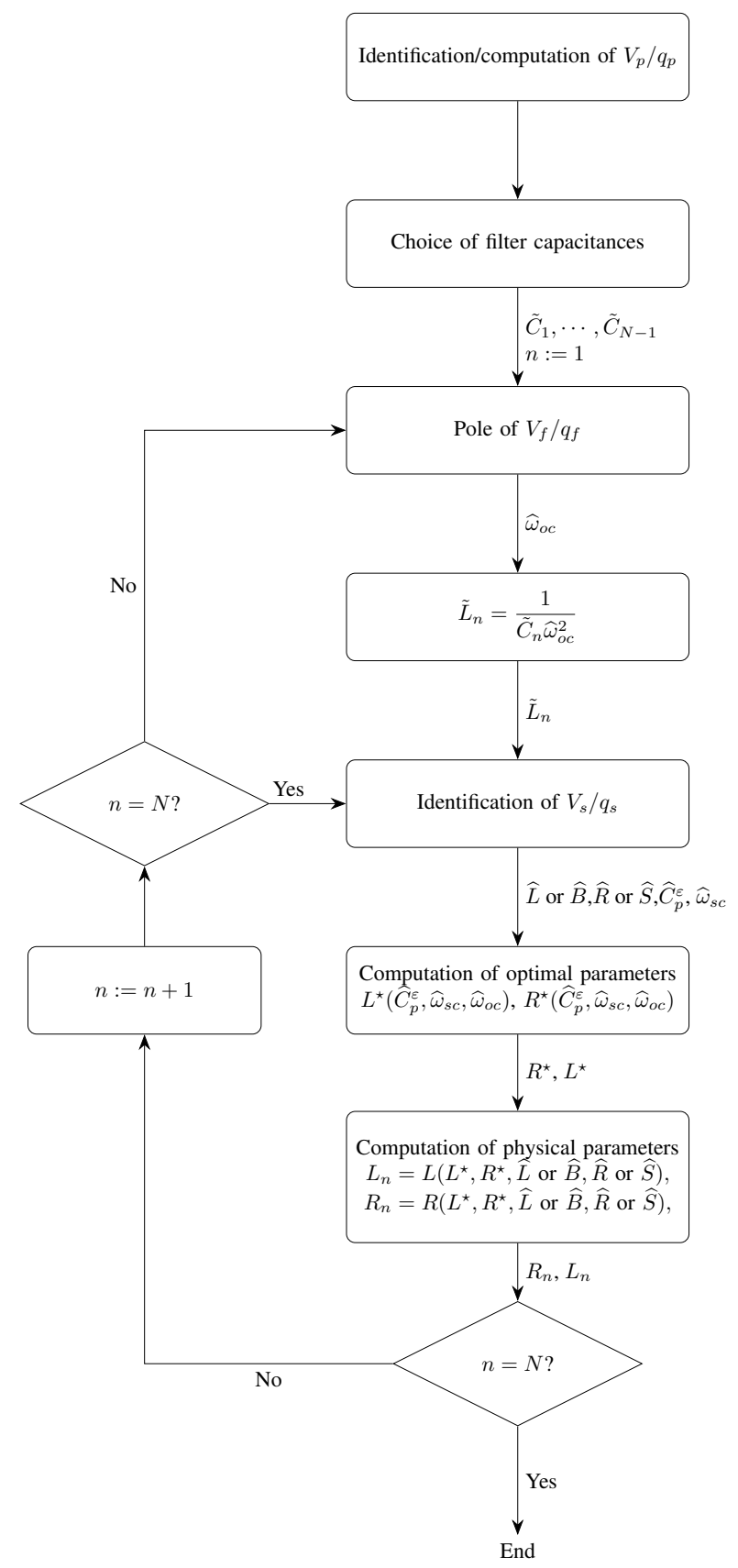

Figure 13. Flowchart of the proposed tuning method.

Filter parameters The current blocking filter parameters are selected such that the filter impedance be infinite at the targeted structural resonance frequency $(\mathrm{Wu}(1998))$. The impedance of the remaining stages associated with higherfrequency modes is therefore negligible compared to that 

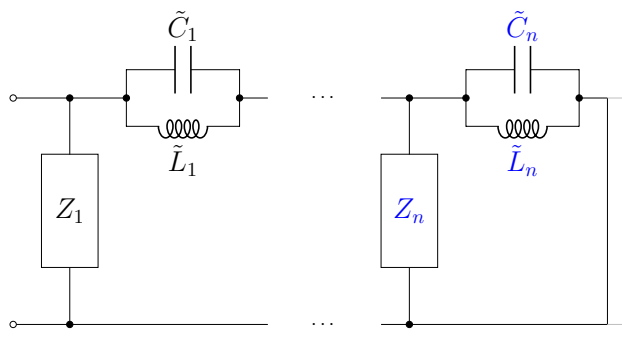

Figure 14. Simplified tuning procedure for stage $n$ : quantities in black are known, quantities in blue are to be tuned, and gray parts are neglected.

of the filter. Thus, these stages may be approximated by a short-circuit, as in Figure 14. This physically-motivated simplification allows to ignore the remaining unknown parameters in the circuit, which drastically simplifies the problem.

The resonance of the current blocking filter at one frequency sets one condition on $\tilde{L}_{n}$ or $\tilde{C}_{n}$, leaving the freedom to choose one of these parameters. Owing to the predictable action of a Parallel capacitor and a Series capacitor on the electromechanical coupling, the latter is set arbitrarily in this work. The filter capacitor is placed in parallel with the currently considered shunt branch, and in series with the following stages. This sets a trade-off between the electromechanical coupling with the currently considered mode and that with higher-frequency modes. Choosing a low value for $\tilde{C}_{n}$ gives high control authority on the $n^{\text {th }}$ controlled mode, but deteriorates the authority on controlled modes $n+1$ to $N$. The converse is also true. A quantitative approach to choose this capacitance based on the BEEMCF is illustrated in the Applications.

The transfer function $V_{f} / q_{f}$ is then formed from the network composed of the previous stages and the filter capacitor, as depicted in Figure 15, using Equation (6). Its pole is identified as $\widehat{\omega}_{o c}$; the filter inductance is finally given by

$$
\tilde{L}_{n}=\frac{1}{\tilde{C}_{n} \widehat{\omega}_{o c}^{2}}
$$

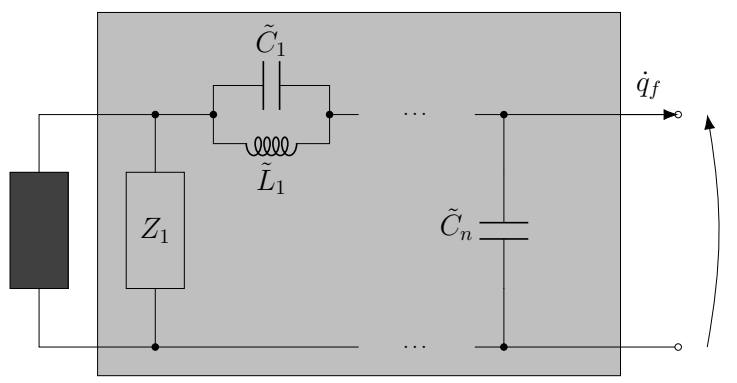

Figure 15. Filter tuning: the black box and light gray box represent the piezoelectric structure and a two-port network, respectively.

Shunt branch parameters The next step is to form a two-port network from an electrical network of known characteristics, constituted of the previous stages and the current blocking filter of the current stage, as shown in Figure 16 (note that the circuits shown in this figure and Figure 14 are topologically equivalent). The optimal parameters of the fictitious shunt circuit may then be derived using the method described in the section Shunt branch connected to an electrical network from which the physical shunt parameters can be calculated.

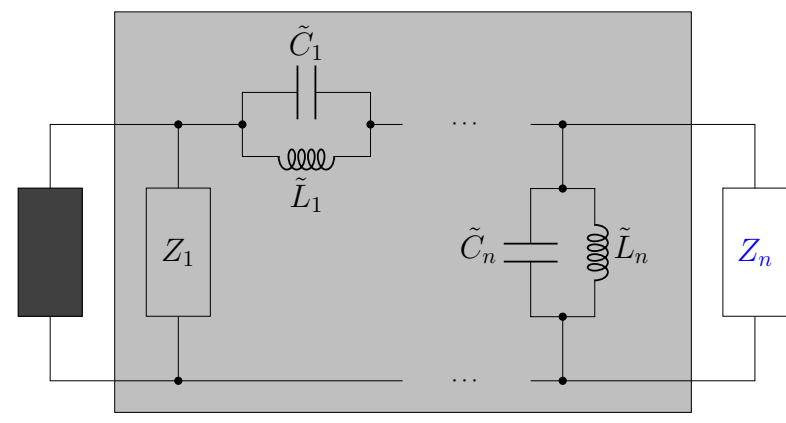

Figure 16. Shunt branch tuning: the black box and light gray box represent the piezoelectric structure and a two-port network, respectively.

Once the four parameters of the considered stage have been determined, the cascade structure is exploited for tuning the next branch of the current blocking shunt circuit. The process is repeated until the last shunt branch $Z_{N}$ has been tuned.

\section{Applications}

In this section, the proposed method is illustrated with numerical and experimental application examples.

\section{A piezoelectric beam}

The piezoelectric beam studied by Thomas et al. (2012) is taken as a first example to demonstrate the method. It is a cantilever beam with two symmetrically-bonded patches connected in series to form the port of a piezoelectric shunt circuit. The finite element model of the beam was built following the method described by Thomas et al. (2009). Proportional damping of $0.1 \%$ was added to the first two modes of the beam. Performance is assessed through the driving-point receptance of the beam transversally excited at its free end (simply referred to as "FRF" hereafter).

Improvement brought by the proposed tuning method A two-mode current blocking shunt circuit with parallel RL branches is connected to the piezoelectric patches. The filter capacitance $\tilde{C}$ is chosen equal to $C_{p}^{\varepsilon}$. The circuit is tuned according to three different methods, namely those proposed in $\mathrm{Wu}(1998)$ and in Agneni et al. (2006) (adapted for parallel RL shunt branches) and the present method.

\begin{tabular}{cccc} 
Parameter & $\mathrm{Wu}(1998)$ & Agneni et al. $\sqrt{(2006)}$ & This work \\
\hline$R_{1}(\Omega)$ & $1,408,917$ & $1,408,917$ & 973,609 \\
$L_{1}(\mathrm{H})$ & 589.34 & 589.34 & 558.35 \\
$\tilde{C}_{1}(\mathrm{nF})$ & 9.16 & 9.16 & 9.16 \\
$\tilde{L}_{1}(\mathrm{H})$ & 570.79 & 570.79 & 576.54 \\
$R_{2}(\Omega)$ & 280,398 & $1,092,542$ & 777,411 \\
$L_{2}(\mathrm{H})$ & 33.48 & 33.28 & 32.52
\end{tabular}

Table 3. Parameters for a two-mode current blocking shunt circuit with parallel $R L$ branches.

Figure 17 compares the obtained FRFs whereas Table 3 lists the corresponding parameters. Wu's and Agneni's 
tuning rules yield unequal resonance peaks because they neglect non-resonant modes (Høgsberg and Krenk 2017). They also ignore the change in coupling caused by the filter capacitance (e.g., the second mode is overdamped with Wu's method). Conversely, the proposed method yields a solution very close from the $H_{\infty}$-optimal solution.

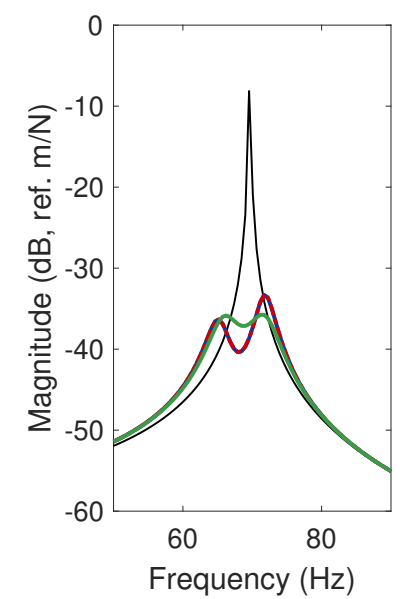

(a)

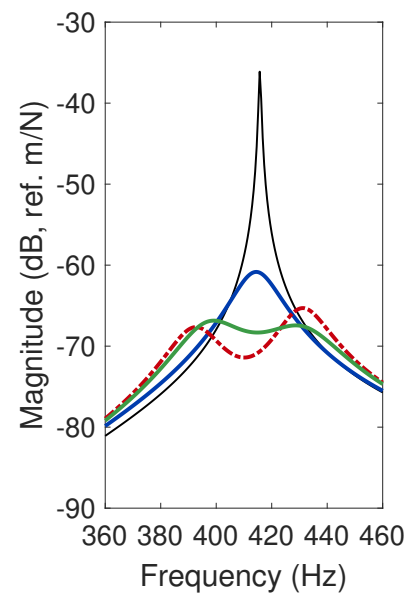

(b)
Figure 17. FRF of the beam with open-circuited patches (-) and with a two-mode current blocking shunt circuit ( - :Wu (1998), ---:Agneni et al. 2006, - : proposed method): mode 1 (a) and mode 2[(b)

To check the validity of the approximations made throughout this work, the dynamic elastance seen from the first shunt branch is analyzed in Figure 18. The elastance obtained when assuming that the second shunt branch is a short circuit is in excellent agreement with the true elastance obtained after tuning the whole current blocking shunt circuit. In addition, the fitted elastance is also a close approximation to the other elastances. Figure 19 shows the dynamic elastance seen from the second shunt branch and its fitted approximation. All these results justify the approximations made to obtain Equation (9) and (11).

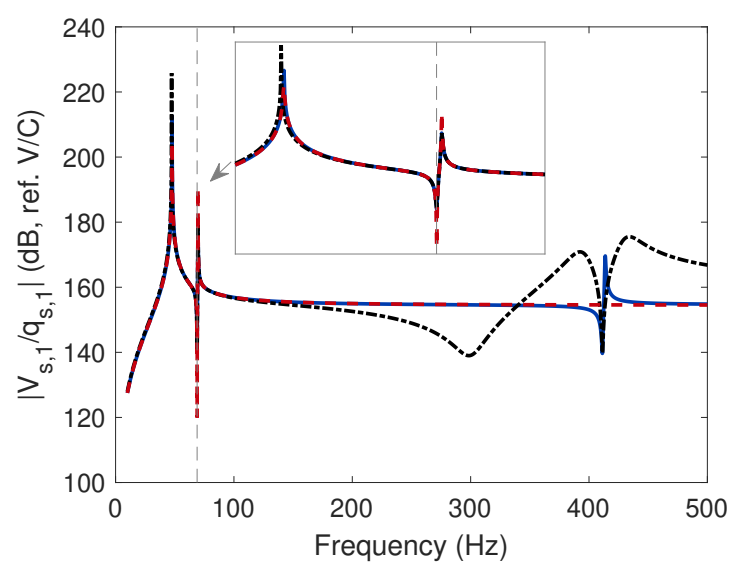

Figure 18. Dynamic elastance obtained by neglecting the second shunt branch (-), true dynamic elastance (-.-) and fitted dynamic elastance around the first mode (- -). The frequency $\omega_{s c, 1}$ is given for reference (- -).

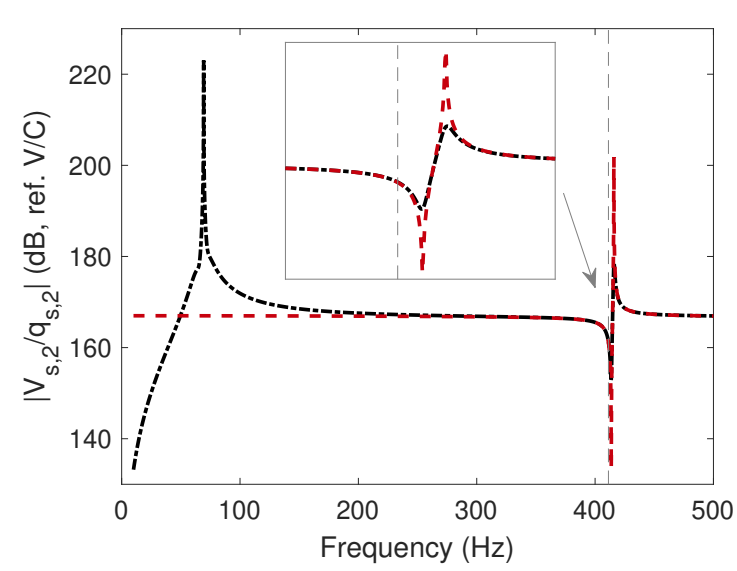

Figure 19. True dynamic elastance (---) and fitted dynamic elastance around the second mode (- $)$. The frequency $\omega_{s c, 2}$ is given for reference (- -).

Filter capacitance effect A two-mode current blocking shunt circuit with series RL shunt branches is now considered. Figure 20 shows the evolution of the BEEMCF when the filter capacitance is varied. The aforementioned trade-off is clearly visible, i.e., a small (large) capacitance offers a good coupling with mode 1 (2).

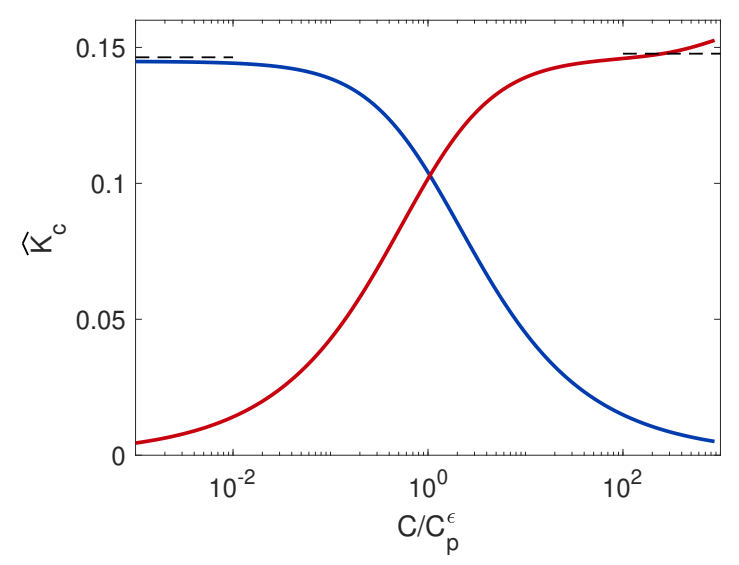

Figure 20. Evolution of the BEEMCF with the first $(-)$ and second $(-)$ mode of the beam. Thin black dashed lines indicate the nominal EEMCFs with mode 1 (left) and 2 (right).

Figure 21 compares the vibration attenuation brought by shunt damping on both modes. This attenuation was computed in two ways, namely directly from the FRF and with the formula given by Thomas et al. (2012) where the BEEMCFs replace the EEMCFs. The two approaches give similar results, thereby supporting the relevance of the BEEMCFs. They can even be used in conjunction with the formula of Thomas et al. (2012) to give an approximate measure of the expected attenuation to guide quantitatively the choice of filter capacitances.

Control of multiple modes A final numerical investigation is carried out by designing a current blocking shunt circuit with series RL shunt branches aiming to control the first four bending modes of the beam. For conciseness, only the case where all filter capacitances are equal to the piezoelectric capacitance at constant strain is discussed. Table 4 lists the 


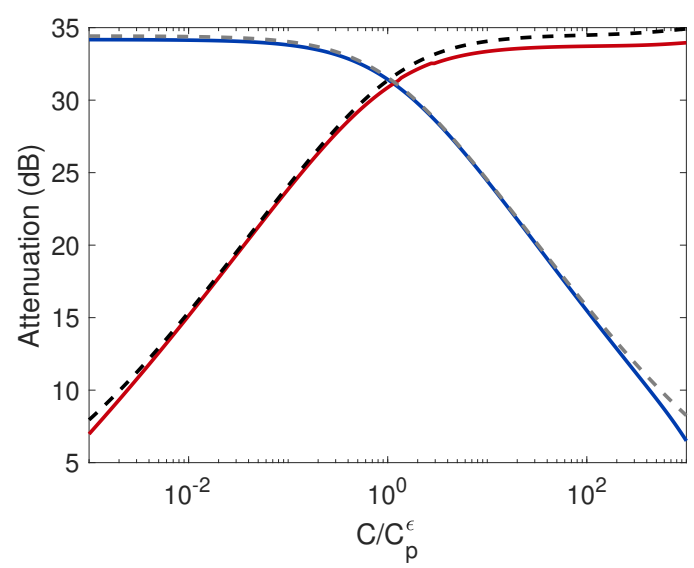

Figure 21. Evolution of the attenuation with the first (-: direct computation, - -: formula from Thomas et al. (2012)) and second (- direct computation, - -: formula from Thomas et al. (2012)) mode of the beam.

resulting parameters. Figure 22 evidences that the proposed method offers an appreciable vibration reduction for the four targeted modes.

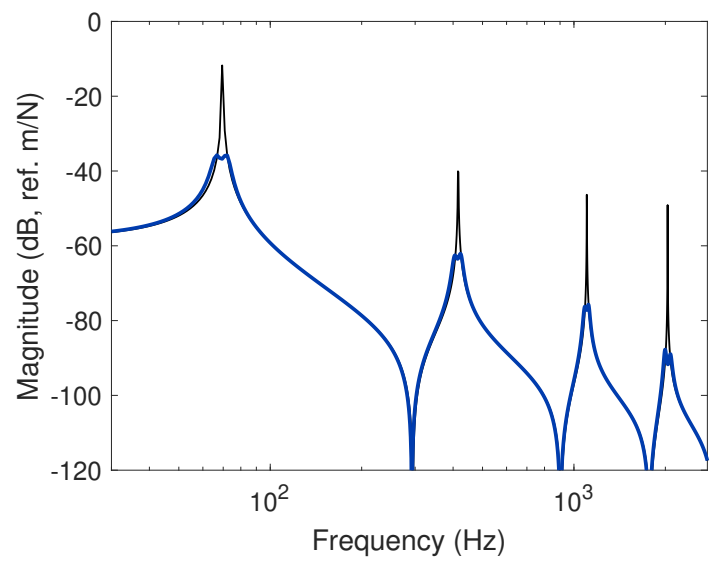

Figure 22. FRF of the beam with open-circuited patches (-) and controlled by a four-mode current blocking shunt circuit (-).

\begin{tabular}{ccccc} 
Stage number $n$ & $R_{n}(\Omega)$ & $L_{n}(\mathrm{H})$ & $\tilde{C}_{n}(\mathrm{nF})$ & $\tilde{L}_{n}(\mathrm{H})$ \\
\hline 1 & 57,322 & 525.17 & 9.16 & 576.53 \\
2 & 18,202 & 31.42 & 9.16 & 16.11 \\
3 & 11,098 & 7.69 & 9.16 & 2.28 \\
4 & 1,609 & 3.78 & $/$ & $/$
\end{tabular}

Table 4. Parameters of the four-mode current blocking shunt circuit

\section{Experimental validation}

The experimental set-up in Figure 23 comprises a cantilever beam covered with an array of ten piezoelectric cells, each cell corresponding to a pair of two stacked PZT-54A piezoelectric patches placed on either side of the beam. A clamped thin lamina is attached to the free end of the beam. More information about the experimental set-up can be found in Lossouarn et al. (2018).

The structure was excited by an electrodynamic shaker, and an impedance head was used to monitor its dynamic response. The excitation level was kept low enough so as to avoid any nonlinear behavior. The two cells closest to the clamped end of the beam were connected in parallel to form one pole of the port of the piezoelectric shunt circuit, the other pole being the line grounded to the beam.

Resonant shunt circuits may require a large inductance for operation with low-frequency modes, typically from hundreds to thousands of Henries, which is largely above what is commercially available. This issue can be solved using active means such as virtual inductors (Park and Inman 2003) and synthetic impedances (Fleming et al.|2000), or using fully passive tailor-made inductors (Lossouarn et al. 2017). In this study, a digital absorber was used to emulate different shunt circuits: either a single-mode series RL circuit or a two-mode current blocking shunt circuit with series RL shunt branches. The absorber enforces through its analog circuitry an arbitrary voltage-to-current relation by programming its digital unit. A sampling frequency of $10 \mathrm{kHz}$ was chosen, and delay-induced destabilization was compensated for using the method proposed in Raze et al. (2019).

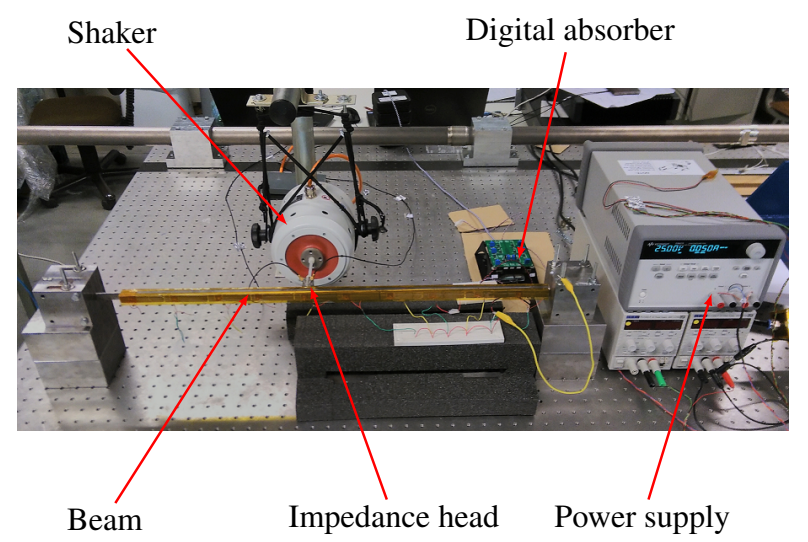

Figure 23. Experimental set-up.

The inputs for the proposed approach can easily be obtained experimentally. The piezoelectric capacitance at constant strain was measured with a multimeter. The resonance frequencies of the first and second bending modes of the beam with short- and open-circuited patches were obtained through simple identification of the corresponding FRFs. The identified parameters are gathered in Table 5 No sophisticated model of the structure was required to tune the shunt circuits. Equations (4) and (27) were used to obtain an experimental transfer function $V_{p} / q_{p}$ from the data in Table 5 (modal damping ratios were assumed to be zero, in view of the lightly-damped character of the host structure). Once a particular value for the filter capacitance was chosen, the remainder of the tuning procedure was carried out by implementing the method outlined in Figure 13 The resulting electrical parameters of the shunt circuit were used to emulate the latter with the digital absorber.

Figure 24 presents experimental FRFs obtained with different electrical boundary conditions on the patches. In particular, the FRFs corresponding to a single-mode piezoelectric shunt circuit show that an attenuation of $21 \mathrm{~dB}$ and $22 \mathrm{~dB}$ can be obtained for mode 1 and 2, respectively.

Figure 25 displays experimental FRFs of the structure controlled by a two-mode current blocking shunt circuit with 


\begin{tabular}{cl} 
Parameter & Value \\
\hline$f_{s c, 1}(\mathrm{~Hz})$ & 30.43 \\
$f_{o c, 1}(\mathrm{~Hz})$ & 30.61 \\
$f_{s c, 2}(\mathrm{~Hz})$ & 144.95 \\
$f_{o c, 2}(\mathrm{~Hz})$ & 145.21 \\
$C_{p}^{\varepsilon}(\mathrm{nF})$ & 103
\end{tabular}

Table 5. Identified parameters of the experimental setup.

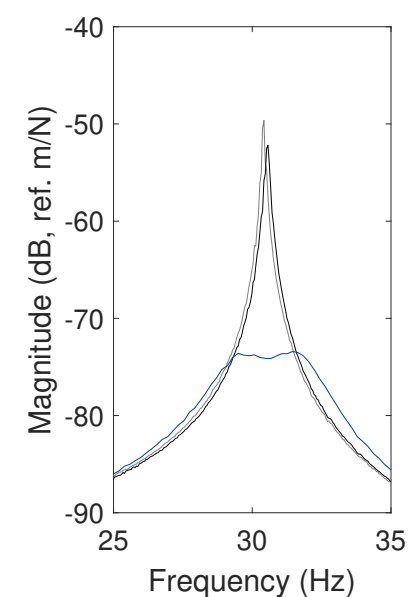

(a)

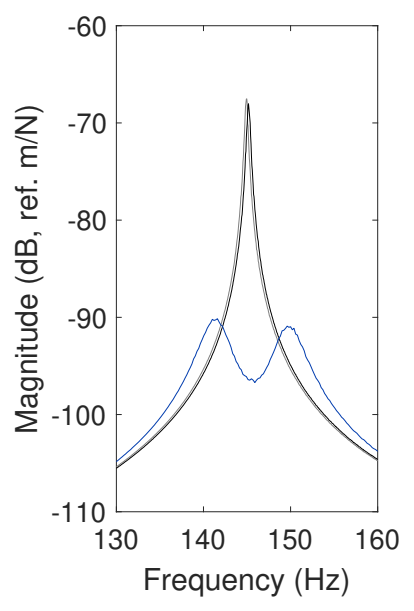

(b)
Figure 24. FRF of the experimental beam with short-circuited patches $(-)$, open-circuited patches $(-)$ and with a single-mode piezoelectric shunt (-). Mode 1 (a) or mode 2 (b)

various values of the filter capacitance. Clearly, the designed circuit is able to provide effective, simultaneous vibration mitigation of the two targeted modes.

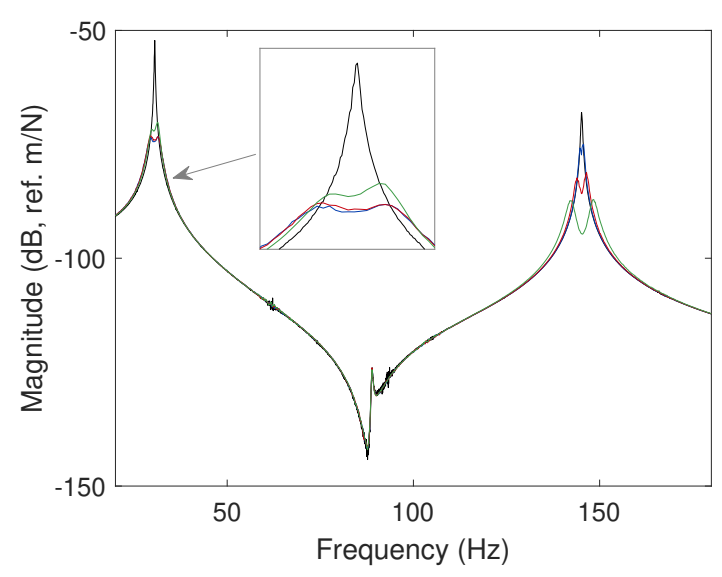

Figure 25. FRF of the experimental beam with open-circuited patches (-) and with a two-mode current blocking shunt circuit: $\tilde{C} / C_{p}^{\varepsilon}=10^{-2}(-), \tilde{C} / C_{p}^{\varepsilon}=10^{-1}(-)$ and $\tilde{C} / C_{p}^{\varepsilon}=1(-)$.

\section{Conclusion}

This paper develops a tuning approach for a simplified current blocking shunt circuit which, when connected to a piezoelectric transducer bonded to a structure, can provide multimodal damping. The case of a general shunt branch connected to an electrical network was first studied with the two-port network formalism. A procedure accounting for the surrounding electrical elements and changes in electromechanical coupling was established. The current blocking shunt circuit was then simplified to be representable by a two-port network of known characteristics. The tuning procedure of each stage was finally detailed, including a discussion on the effect of the arbitrary choice of the filter capacitance. It should be noted that this procedure is theoretically independent of the two-port network formalism adopted herein, but the versatility of the latter makes it an attractive modelling framework, as testified by the simple expressions for the transfer functions involved.

The procedure was numerically demonstrated using a piezoelectric beam example from the literature; it brought noticeable improvements with respect to existing tuning rules. It was also shown that the shunt circuit can control an arbitrary number of modes. Eventually, the approach was validated experimentally.

\section{Acknowledgements}

The authors would like to acknowledge the financial support of the SPW (WALInnov grant 1610122) and Valery Broun for his help in building the digital vibration absorber.

\section{Appendix}

\section{Series $R L$ shunt tuning}

The optimal characteristics of a series RL shunt circuit can be computed from the knowledge of $C_{p}^{\varepsilon}, K_{c}$ and $\omega_{o c}$. Introducing

$$
r=\frac{\sqrt{64-16 K_{c}^{2}-26 K_{c}^{4}}-K_{c}^{2}}{8}
$$

the optimal inductance $L$ and resistance $R$ are given by

$$
L=\frac{4 K_{c}^{2}+4}{3 K_{c}^{2}-4 r+8} \frac{1}{\omega_{o c}^{2} C_{p}^{\varepsilon}}
$$

and

$$
\begin{gathered}
R=\frac{2 \sqrt{2\left(K_{c}^{2}+1\right)\left(27 K_{c}^{4}+K_{c}^{2}(80-48 r)-64(r-1)\right)}}{\left(5 K_{c}^{2}+8\right) \sqrt{3 K_{c}^{2}-4 r+8}} \\
\frac{1}{\omega_{o c} C_{p}^{\varepsilon}}, \quad \text { (38) }
\end{gathered}
$$

respectively ; see Soltani et al. (2014); Ikegame et al. (2019) for details.

\section{Parallel RL shunt tuning}

The analytical $H_{\infty}$ solution was found by Tang et al. (2016) for an electromagnetic transducer shunted with a series RC circuit. As shown by Ikegame et al. (2019), this problem is equivalent to a piezoelectric transducer shunted with a parallel RL shunt. The optimal formulae are then obtained 
by first computing the series of coefficients

$$
\begin{aligned}
& b_{0}=64 \\
& b_{1}=-16 K_{c}^{2} \\
& b_{2}=-64+16 K_{c}^{2}+11 K_{c}^{4} \\
& b_{3}=2 K_{c}^{2}\left(8-K_{c}^{2}\right)\left(2-K_{c}^{2}\right) \\
& b_{4}=-2 K_{c}^{4}\left(2-K_{c}^{2}\right) \\
& a_{6}=27\left(b_{0} b_{3}^{2}+b_{1}^{2} b_{4}\right)-9 b_{2}\left(b_{1} b_{3}+8 b_{0} b_{4}\right)+2 b_{2}^{3} \\
& a_{5}=12 b_{0} b_{4}-3 b_{1} b_{3}+b_{2}^{2} \\
& a_{4}=\sqrt[3]{\frac{\sqrt{a_{6}^{2}-4 a_{5}^{3}}+a_{6}}{2}} \\
& a_{3}=\frac{1}{2} \sqrt{\frac{b_{1}^{2}}{b_{0}^{2}}+\frac{4\left(a_{4}^{2}+a_{5}-2 b_{2} a_{4}\right)}{3 b_{0} a_{4}}} \\
& a_{2}=-\frac{8 b_{0}^{2} b_{3}-4 b_{0} b_{1} b_{2}+b_{1}^{3}}{4 b_{0}^{3} a_{3}} \\
& a_{1}=\frac{3 b_{1}^{2} a_{4}-2 b_{0}\left(4 b_{2} a_{4}+a_{4}^{2}+a_{5}\right)}{6 b_{0}^{2} a_{4}}
\end{aligned}
$$

and finally,

$$
r=-\frac{b_{1}}{4 b_{0}}+\frac{a_{3}}{2}+\frac{\sqrt{a_{1}+a_{2}}}{2}
$$

and the optimal inductance $L$ and resistance $R$ are given by

$$
L=\frac{6}{2 r-K_{c}^{2}+\sqrt{16 r^{2}-4 r K_{c}^{2}+K_{c}^{4}}} \frac{1}{\omega_{s c}^{2} C_{p}^{\varepsilon}}
$$

and

$$
R=\frac{1}{2} \sqrt{r \frac{2 r+K_{c}^{2}-\sqrt{16 r^{2}-4 r K_{c}^{2}+K_{c}^{4}}}{(r-1)\left(3 r-2 K_{c}^{2}\right)}} \sqrt{\frac{L}{C_{p}^{\varepsilon}}}
$$

respectively ; see Tang et al. (2016); Ikegame et al. (2019) for details.

\section{References}

Agneni A, Del Sorbo M, Mastroddi F and Polli GM (2006) Multi-modal damping by shunted piezo-patches: Possible aeroelastic applications. International Journal of Applied Electromagnetics and Mechanics 24(1-2): 1-24. DOI:10.3233/JAE-2006-709. URL https: //www.medra.org/servlet/aliasResolver? alias=iospress\&doi=10.3233/JAE-2006-709

Alessandroni S, Dell'Isola F and Porfiri M (2002) A revival of electric analogs for vibrating mechanical systems aimed to their efficient control by PZT actuators. International Journal of Solids and Structures 39(20): 5295-5324. DOI:10.1016/S0020-7683(02)00402-X. URL https://linkinghub.elsevier.com/retrieve/ pii/S002076830200402X.

Alexander CK and Sadiku MNO (2000) Fundamentals of Electric Circuits. Cambridge: McGraw-Hill Education. ISBN 0073380571.

Behrens S, Moheimani S and Fleming A (2003) Multiple mode current flowing passive piezoelectric shunt controller. Journal of Sound and Vibration 266(5): 929-942. DOI:10.1016/S0022-460X(02)01380-9. URL https://linkinghub.elsevier.com/retrieve/ pii/s0022460X02013809.
Berardengo M, Manzoni S and Conti AM (2017) Multi-mode passive piezoelectric shunt damping by means of matrix inequalities. Journal of Sound and Vibration 405: 287-305. DOI:10.1016/j.jsv.2017.06.002. URL http://dx.doi. org/10.1016/j.jsv.2017.06.002.

Berardengo M, Manzoni S, Thomas O and Vanali M (2018) Piezoelectric resonant shunt enhancement by negative capacitances: Optimisation, performance and resonance cancellation. Journal of Intelligent Material Systems and Structures 29(12): 2581-2606. DOI:10.1177/1045389X18770874. URL https : //doi.org/10.1177/1045389x18770874

Berardengo M, Thomas O, Giraud-Audine $\mathrm{C}$ and Manzoni S (2016) Improved resistive shunt by means of negative capacitance: new circuit, performances and multimode control. Smart Materials and Structures 25(7): 075033. DOI:10.1088/0964-1726/25/7/075033. URL https://iopscience.iop.org/article/10. 1088/0964-1726/25/7/075033/meta

Caruso G (2001) A critical analysis of electric shunt circuits employed in piezoelectric passive vibration damping. Smart Materials and Structures 10(5): 1059-1068. DOI: 10.1088/0964-1726/10/5/322. URL http://stacks. iop.org $/ 0964-1726 / 10 / i=5 / \mathrm{a}=322$ ? $\mathrm{key}=$ crossref.c36301d22f58c73ce0c4983b6cc0ce51

Cigada A, Manzoni S, Redaelli M and Vanali M (2012) Optimization of the current flowing technique aimed at semi-passive multi-modal vibration reduction. Journal of Vibration and Control 18(2): 298-312. DOI:10. 1177/1077546311407537. URL http://journals. sagepub.com/doi/10.1177/1077546311407537

de Marneffe B (2007) Active and Passive Vibration Isolation and Damping via Shunted Transducers. $\mathrm{PhD}$ Thesis, Universite Libre de Bruxelles. URL http://hdl.handle.net/2013/ULB-DIPOT: oai:dipot.ulb.ac.be:2013/210613

Edberg DL, Bicos AS and Fechter JS (1991) On piezoelectric energy conversion for electronic passive damping enhancement. In: Proceedings of Damping. pp. pp. 717-724.

Fleming A, Behrens S and Moheimani S (2000) Synthetic impedance for implementation of piezoelectric shunt-damping circuits. Electronics Letters 36(18): 1525. DOI:10.1049/el:20001083. URL https: //digital-library.theiet.org/content/ journals/10.1049/el_20001083

Fleming A, Behrens S and Reza Moheimani S (2002) Optimization and implementation of multimode piezoelectric shunt damping systems. IEEE/ASME Transactions on Mechatronics 7(1): 87-94. DOI:10.1109/3516.990891. URL http:// ieeexplore.ieee.org/document/990891/

Fleming AJ, Behrens S and Moheimani SOR (2003) Reducing the inductance requirements of piezoelectric shunt damping systems. Smart Materials and Structures 12(1): 57-64. DOI: 10.1088/0964-1726/12/1/307. URL http://stacks. iop.org/0964-1726/12/i=1/a=307? key= crossref.39ab51d3ef8ca1b6552bf22dd04b4ff3

Gardonio P and Casagrande D (2017) Shunted piezoelectric patch vibration absorber on two-dimensional thin structures: Tuning considerations. Journal of Sound and Vibration 395: 2647. DOI:10.1016/j.jsv.2017.02.019. URLhttp://dx. doi. org/10.1016/j.jsv.2017.02.019. 
Giorgio I, Galantucci L, Della Corte A and Del Vescovo D (2015) Piezo-electromechanical smart materials with distributed arrays of piezoelectric transducers: Current and upcoming applications. International Journal of Applied Electromagnetics and Mechanics 47(4): 10511084. DOI:10.3233/JAE-140148. URL https: //www.medra.org/servlet/aliasResolver? alias=iospress\&doi=10.3233/JAE-140148

Goldstein AL (2011) Self-Tuning Multimodal Piezoelectric Shunt Damping. Journal of the Brazilian Society of Mechanical Sciences and Engineering 33(4): 428436. DOI:10.1590/S1678-58782011000400006. URL http://www.scielo.br/scielo.php?script = sci_arttext\&pid=S1678-58782011000400006\& lng=en\&nrm=iso\&t lng=en

Guo KM and Jiang J (2014) Independent modal resonant shunt for multimode vibration control of a truss-cored sandwich panel. International Journal of Dynamics and Control 2(3): 326-334. DOI:10.1007/s40435-013-0036-7. URL http://link. springer.com/10.1007/s40435-013-0036-7

Hagood N and von Flotow A (1991) Damping of structural vibrations with piezoelectric materials and passive electrical networks. Journal of Sound and Vibration 146(2): 243-268. DOI:10.1016/0022-460X(91)90762-9. URL https://linkinghub.elsevier.com/retrieve/ pii/0022460X91907629.

Hagood NW, Chung WH and von Flotow A (1990) Modelling of Piezoelectric Actuator Dynamics for Active Structural Control. Journal of Intelligent Material Systems and Structures 1(3): 327-354. DOI:10.1177/1045389X9000100305. URL http://journals.sagepub.com/doi/10.1177/ $1045389 \times 9000100305$

Høgsberg J and Krenk S (2017) Calibration of piezoelectric RL shunts with explicit residual mode correction. Journal of Sound and Vibration 386: 65-81. DOI:10.1016/j.jsv.2016. 08.028. URL http://dx.doi.org/10.1016/j.jsv. 2016.08 .028

Hollkamp JJ (1994) Multimodal Passive Vibration Suppression with Piezoelectric Materials and Resonant Shunts. Journal of Intelligent Material Systems and Structures 5(1): 49-57. DOI:10.1177/1045389X9400500106. URL http://journals.sagepub.com/doi/10.1177/ $1045389 \times 9400500106$

Ikegame T, Takagi K and Inoue T (2019) Exact Solutions to $\mathrm{H} \infty$ and H 2 Optimizations of Passive Resonant Shunt Circuit for Electromagnetic or Piezoelectric Shunt Damper. Journal of Vibration and Acoustics 141(3): 031015. DOI:10.1115/ 1.4042819. URL http://vibrationacoustics. asmedigitalcollection.asme.org/article. aspx?doi=10.1115/1.4042819.

Jeon JY (2009) Passive vibration damping enhancement of piezoelectric shunt damping system using optimization approach. Journal of Mechanical Science and Technology 23(5): 1435-1445. DOI:10.1007/s12206-009-0402-8. URL http://link.springer.com/10.1007/ s12206-009-0402-8.

Lossouarn B, Aucejo M, Deü JF and Multon B (2017) Design of inductors with high inductance values for resonant piezoelectric damping. Sensors and Actuators A: Physical 259: $68-76$.

DOI:10.1016/j.sna.2017.03.030. http://dx.doi.org/10.1016/j.sna.2017.

03.030https://linkinghub.elsevier.com/ retrieve/pii/s0924424716309785

Lossouarn B, Deü JF, Aucejo M and Cunefare KA (2016) Multimodal vibration damping of a plate by piezoelectric coupling to its analogous electrical network. Smart Materials and Structures 25(11): $115042 . \quad$ DOI: 10.1088/0964-1726/25/11/115042. URL http://stacks. iop.org $/ 0964-1726 / 25 / i=11 / a=115042$ ? key= crossref.336b6c7b8578169e34455e93e5f8b4b8

Lossouarn B, Deü JF and Kerschen G (2018) A fully passive nonlinear piezoelectric vibration absorber. Philosophical Transactions of the Royal Society A: Mathematical, Physical and Engineering Sciences 376(2127): 20170142. DOI:10.1098/rsta.2017.0142. URL http://rsta.royalsocietypublishing.org/ lookup/doi/10.1098/rsta.2017.0142.

Moheimani SOR and Fleming AJ (2006) Piezoelectric Transducers for Vibration Control and Damping. Advances in Industrial Control. London: Springer-Verlag. ISBN 1-84628-331-0. DOI: 10.1007/1-84628-332-9. URL http: / / ink.springer. $\mathrm{com} / 10.1007 / 1-84628-332-9$

Park CH and Inman DJ (2003) Enhanced Piezoelectric Shunt Design. Shock and Vibration 10(2): 127-133. DOI:10. 1155/2003/863252. URL http://wWw.hindawi.com/ journals/sv/2003/863252/abs/

Raze G, Paknejad A, Zhao G, Broun V and Collette C (2019) Suppression of delay-induced instabilities of digital piezoelectric vibration absorbers. In: Benjeddou A, Mechbal N and Deü JF (eds.) Proceedings of the 9th ECCOMAS Thematic Conference on Smart Structures and Materials - SMART 2019. International Centre for Numerical Methods in Engineering (CIMNE), pp. 991-1001. URL http://hdl . handle. net/2268/240342

Soltani P, Kerschen G, Tondreau G and Deraemaeker A (2014) Piezoelectric vibration damping using resonant shunt circuits: an exact solution. Smart Materials and Structures 23(12): $125014 . \quad$ DOI: 10.1088/0964-1726/23/12/125014. URL http: //stacks . iop.org $/ 0964-1726 / 23 / \mathrm{i}=12 / \mathrm{a}=125014$ ? key= crossref.798743ce34b627bed945b046d8a6b51a

Tang X, Liu Y, Cui W and Zuo L (2016) Analytical Solutions to H2 and $\mathrm{H} \infty$ Optimizations of Resonant Shunted Electromagnetic Tuned Mass Damper and Vibration Energy Harvester. Journal of Vibration and Acoustics 138(1). DOI:10.1115/1.4031823. URL https://asmedigitalcollection. asme.org/vibrationacoustics/article/ doi/10.1115/1.4031823/472483/

Analytical-Solutions-to-H2-and-H-Optimizations-of

Thomas O, Deü JF and Ducarne J (2009) Vibrations of an elastic structure with shunted piezoelectric patches: efficient finite element formulation and electromechanical coupling coefficients. International Journal for Numerical Methods in Engineering 80(2): 235-268. DOI:10.1002/nme.2632. URL http://doi.wiley.com/10.1002/nme.2632

Thomas O, Ducarne J and Deü JF (2012) Performance of piezoelectric shunts for vibration reduction. Smart Materials and Structures 21(1): $015008 . \quad$ DOI: 10.1088/0964-1726/21/1/015008. URL http: / / stacks . iop.org/0964-1726/21/i=1/a=015008? key= 
crossref.6d8696aab65a8889d18c15289623f766

Toftekær JF, Benjeddou A, Høgsberg J and Krenk S (2018)

Optimal piezoelectric resistive-inductive shunt damping of plates with residual mode correction. Journal of Intelligent Material Systems and Structures 29(16): 3346-3370. DOI: 10.1177/1045389X18798953. URL http://journals. sagepub.com/doi/10.1177/1045389X18798953

Toftekær JF and Høgsberg J (2020) Multi-mode piezoelectric shunt damping with residual mode correction by evaluation of modal charge and voltage. Journal of Intelligent Material Systems and Structures 31(4): 570-586. DOI: 10.1177/1045389X19891646. URL http://journals. sagepub.com/doi/10.1177/1045389X19891646

Viana FAC and Steffen, Jr V (2006) Multimodal vibration damping through piezoelectric patches and optimal resonant shunt circuits. Journal of the Brazilian Society of Mechanical Sciences and Engineering 28(3): 293310. DOI:10.1590/S1678-58782006000300007. URL http://www.scielo.br/scielo.php?script= sci_arttext\&pid=S1678-58782006000300007\& lng=en\&nrm=iso\&t lng=en

Wu SY (1996) Piezoelectric shunts with a parallel R-L circuit for structural damping and vibration control. In: Johnson CD (ed.) Spie, volume 2720. ISBN 0819420956, pp. 259-269. DOI:10.1117/12.239093. URL http://proceedings.spiedigitallibrary. org/proceeding.aspx?articleid=1017503

Wu SY (1998) Method for multiple-mode shunt damping of structural vibration using a single PZT transducer. In: Davis LP (ed.) Smart structures and materials 1998: passive damping and isolation, volume 3327. pp. 159-168. DOI:10.1117/12.310680. URL http: //proceedings.spiedigitallibrary. org/proceeding.aspx?articleid=940157 